\title{
Numerical aided design of Pelton nozzle jet deflector
}

Boro POPOVSKI, Andrej LIPEJ

DOI: 10.30464/jmee.2021.5.2.149

Cite this article as:

Popovski B., Lipej A. Numerical aided design of Pelton nozzle jet deflector. Journal of Mechanical and Energy Engineering, Vol. 5(45), No. 2, 2021, pp. 149-156.

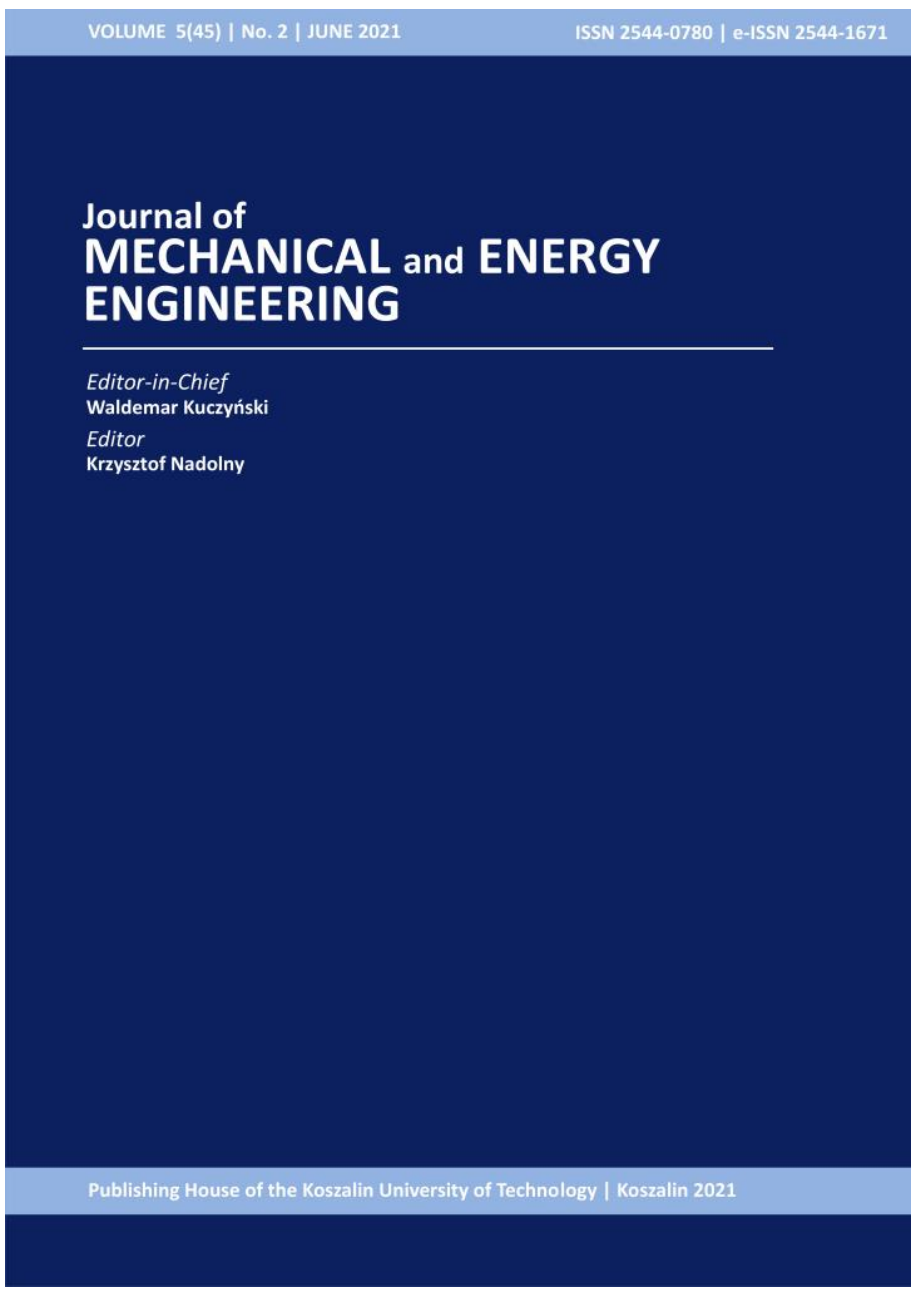

Journal of Mechanical and Energy

Engineering

Website: jmee.tu.koszalin.pl

ISSN (Print): 2544-0780

ISSN (Online): 2544-1671

Volume: 5(45)

Number: 2

Year: 2021

Pages: 149-156

Article Info:

Received 21 April 2021

Accepted 18 May 2021

\section{Open Access}

This article is distributed under the terms of the Creative Commons Attribution 4.0 (CC BY 4.0) International License (http://creativecommons.org/licenses/by/4.0/), which permits unrestricted use, distribution, and reproduction in any medium, provided you give appropriate credit to the original author(s) and the source, provide a link to the Creative Commons license, and indicate if changes were made. 


\title{
NUMERICAL AIDED DESIGN OF PELTON NOZZLE JET DEFLECTOR
}

\author{
Boro POPOVSKI ${ }^{1}$, Andrej LIPEJ ${ }^{2 *}$ \\ ${ }^{1}$ Turbine design $\&$ consulting S.P., Slovenia \\ ${ }^{2}$ Faculty of Mechanical Engineering, University of Novo Mesto, Na Loko 2, 8000 Novo mesto, Slovenia, \\ e-mail: andrej.lipej@fs-unm.si
}

(Received 21 April 2021, Accepted 18 May 2021)

\begin{abstract}
Hydro energy still occupies an important place among renewable energy sources. In special operating conditions, Pelton turbines are irreplaceable and can be used for extremely small hydropower plants and also large hydro power plants. Pelton turbines can operate with high head and relatively small flow rates. In many cases, the height differences of the water are very large. Sometimes, it is necessary to stop the operation of the turbine very quickly, and the consequences of water hammer can be very severe. The part that is responsible for minimizing the consequences of this phenomena is a jet deflector, which can be in two different technical designs. Steps for 3-D geometry definition, pre-processing and post-processing, flow modelling and FEM analysis are presented. In the paper, a new optimized design is presented of a push-out jet deflector shape. Optimization includes a 3-D CFD analysis of free surface flow and a stress analysis. The main goal of the research was to minimize the influence of all the force components on the torque of the deflector servomotor. The final results present the geometry of the deflector with a significant reduction in stresses and deformations. These were achieved with a crucial reduction in the hydrodynamic force and torque.
\end{abstract}

Keywords: water turbine, Pelton turbine, jet deflector, free surface flow, numerical analysis

\section{INTRODUCTION}

Today, in the development process of energy machines, it is very important to pay attention to a long service life of the machine and low maintenance costs. It is necessary to avoid the fatigue [1] problems of material due to unnecessary operating characteristics. For this reason, we undertook a detailed numerical stress analysis of the jet deflector of the Pelton turbine. The exact boundary and initial conditions to carry out an FEM analysis can be obtained by computational fluid dynamics (CFD). Computational Fluid Dynamics is a very useful tool in the projection of all properties of hydraulic machines. In impulse turbines, a very complex flow analysis of the multiple phase flow is a major problem. The relative results of energetic and cavitation characteristics are usually very well foreseen. In some cases, the calculation of the absolute results of all the turbine characteristics can still pose a challenge. In this research, we considered a case where the results of numerical analyses using stationary models can be a useful tool for a successful optimization of individual parts of a Pelton turbine.

A numerical analysis of a multiphase nonstationary turbulent flow is a very demanding task in all respects. In order to satisfy all the criteria regarding the accuracy of the calculation, in most cases it is necessary to have very high-quality computational networks with a huge number of elements. As a result, this means very long computing times or the use of very powerful multiple processor computers. Altogether, it may make the development time of an individual new product more expensive or longer.

Therefore, it is always necessary to look for certain simplifications that do not affect the quality of the final result too much. Such an approach is reliable, considering the experience of already successfully completed projects and considering the good practices presented in various articles published at international scientific conferences and in international scientific journals. 
The novelty of the approach presented is the use of a relatively simple numerical method which offers fairly reliable results. Validation of the numerical simulations has been previously performed on various cases.

In the last two decades, many technical and scientific papers concerning the abovementioned problems of flow in Pelton turbines have been presented. Zhang et al. presented a water jet formation from a nozzle based on measurement results and a CFD analysis [2]. The formation of a secondary flow field in front of the nozzle exit and the influence of the velocity distribution inside the jet on the turbine characteristics were analyzed using numerical and experimental methods [3, 4]. In [5] and [6] a two phase flow inside the Pelton turbine runner calculated. Paper [7] analyzed transient phenomena in the Pelton bucket with an CFD analysis. All the results were compared considering pressure distribution measurements on the runner walls. The most detailed investigation of the flow conditions in a Pelton turbine was performed in [8]. Flow conditions were analyzed by measuring the time distribution of pressure along the rotor walls using various visualization methods and by means of numerical calculations. An extensive numerical and experimental analysis of the flow conditions in the Pelton turbine is provided in the doctoral dissertation [9].

Research in recent years has focused mainly on an analysis of the jet quality at the entrance to the rotor $[10,11]$. In a detailed analysis of scientific papers in this field, we obtained a lot of information about the flow conditions and methods for the quality of research in Pelton turbines but we did not find any research concerning the analysis and design of deflector.

In practice, Pelton turbines are machines that operate in extreme conditions. High water drops mean high water speeds, high pressures and forces and consequently high torques that need to be controlled using different motors. To optimize the entire plant, it is important to minimize all the forces and torques as much as possible. For all the cases, it is not possible to give a single recipe for achieving optimal results; however, a method can be presented by means of which these goals can be achieved. Such a presentation of the process was also the purpose of the presented research.

\section{STRESS ANALYSIS}

For a complete analysis of the preferred Pelton nozzle-deflector, it is certainly necessary to include a strong stress analysis because, as it will be presented in the subsequent calculations, it plays a major role in opening and closing the nozzle deflector. The part of the turbine that is examined in this paper is usually oversized considering both stress and geometry because the price here is a "drop in the sea" compared to the other parts and the turbine in general. Special and detailed research and development would take a lot of time and money. However, this stress analysis shows the opposite, especially when it comes to large (considering their dimensions) and high-head Pelton turbines, where there are high speeds at the exit of the nozzle and therefore heavy loads of the jet-deflector.

The analysis of the stress conditions of the deflector, which is the subject of this investigation, is in the area of high-level problems due to the multidirectional forces and curvature of the surface and position of the deflector.

A detailed description of the criteria for the methodology selection and calculations is provided in the methodology which is based on the hypothesis of specific potential energy for shape deformation, i.e. well known as HMH according the initials of the authors: Huber, von Mises and Hencky.

Under the three-axis stresses conditions, the following formulae for equivalent stresses can be derived:

$$
\begin{gathered}
\sigma_{e k}=\sqrt{\sigma_{1}^{2}+\sigma_{2}^{2}+} \sigma_{3}^{2}-\sigma_{1} \sigma_{2}-\sigma_{2} \sigma_{3}-\sigma_{1} \sigma_{3} \\
\leq \sigma_{d} .
\end{gathered}
$$

The hypotheses for maximal deformation energy for the shape changing is generally is applied in numerous software packages for the calculation of the stress-deformation status using the method of finite elements (volumes).

\section{FREE SURFACE MODELLING}

Compared to other types of water turbines, CFD analysis in Pelton turbines is much more demanding. The reasons include two-phase flow, turbulence and unsteadiness. In some cases, the results can be quite accurate with a steady state analysis but a multi-phase flow cannot be avoided. Computational time to obtain a converge solution is usually quite long.

Free surface flow can be analyzed using a homogeneous or inhomogeneous model. For the applications when two fluids are being mixed and separated, the inhomogeneous model is recommended. In case of Pelton turbines analysis, the homogeneous model is more appropriate. The results obtained with the inhomogeneous model can be slightly better but they require higher computational effort. In this paper, we used the homogeneous model.

The homogeneous model assumes that the quantities transported for the process are the same throughout all the phases:

$$
U_{-} \alpha=U, p_{-} \alpha=p, 1 \leq \alpha \leq N p .
$$

It is therefore sufficient to solve bulk transport equations for shared fields instead of solving individual transport equations [12]:

$$
\frac{\partial \rho}{\partial t}+\nabla \cdot(\rho U)=\sum_{\beta=1}^{N_{p}} \Gamma_{\alpha \beta} .
$$




$$
\begin{gathered}
\frac{\partial}{\partial t}(\rho U)+\nabla \cdot\left(\rho U \otimes U-\mu\left(\nabla U+(\nabla U)^{T}\right)\right) \\
=S_{M}-\nabla p .
\end{gathered}
$$

$\Gamma_{\alpha \beta}$ in Eq. (3) is the mass flow rate per unit of volume from phase $\beta$ to phase $\alpha$. Density and viscosity are calculated from density and viscosity of all the phases in the fluid:

$$
\rho=\sum_{\alpha=1}^{N_{p}} r_{\alpha} \rho_{\alpha}, \mu=\sum_{\alpha=1}^{N_{p}} r_{\alpha} \mu_{\alpha} .
$$

A detailed description of multiphase models and modelling of free surface flows can be found in [11]. The near-wall modelling is based on the wall-function theory as an extension of the Launder and Spalding method. In the logarithmic law region, the near wall tangential velocity is related to the wall-stress $\tau_{\omega}$, by means of a logarithmic function. The equation for the near wall velocity is given by:

$$
u^{+}=\frac{U_{t}}{u_{\tau}}=\frac{1}{k} \ln \left(y^{+}\right)+C .
$$

The definition of the $y^{+}$variable is given by the standard definition of $y^{+}$generally used in CFD modelling.

\section{CFD ANALYSIS}

When optimizing the jet deflector, the first condition is the precise calculation of the shape of the jet coming from the nozzle, under certain operating conditions. In order to enable normal calculation times, the computational domain was reduced to the output part of the nozzle and the surroundings of the deflector. The geometry and the computational domain are presented in Fig. 1. It is necessary to provide precise calculations along the nozzle walls, especially in the area where the jet leaves the nozzle. The density of the mesh in this area has a significant influence on the quality of the numerical shape of the jet. It is also necessary to satisfy the conditions for automatic mesh refinement in places where individual phases, air and water are in contact. The first part of the jet depends only on the shape of the nozzle and the quality of the grid at the exit of the nozzle, while the other part of the jet represents a reflecting jet and it depends on the shape of the deflector and the computational grid along the walls of the deflector and the surrounding area.

In the case of automatic grid refinement, the final size of the grid must be considered, which must be such that the CPU time does not become unmanageable. In our case, the refinement was limited to the upper limit of about 20 million elements.

Beside the grid refinement close to the water-air boundary, the special attention was also paid to the quality of the grid near the walls (the values of $y^{+}$). A numerical analysis of the flow in the Pelton turbine deflector was carried out using steady state numerical methods that utilize the turbulent model $k-\omega$ SST.

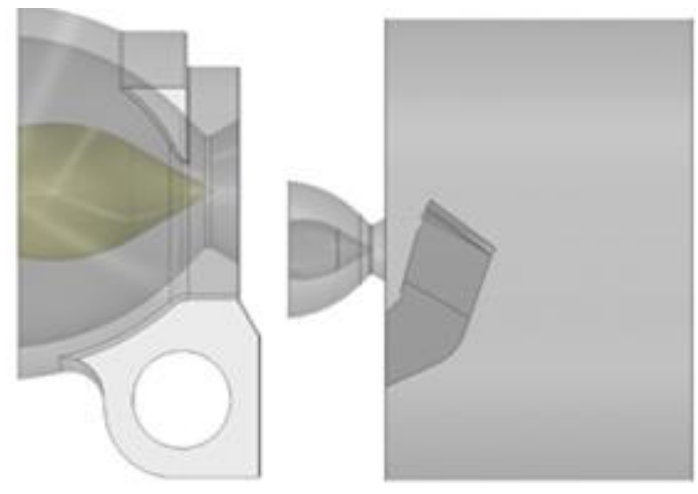

Fig. 1. Geometry of nozzle (left) and computational domain (right)

The results of the numerical analyses for different geometries were compared. We focused on the distribution of the velocity, pressure, and the distribution of the dimensionless parameter $y+$. The quality and convergence of the results for refined computational grids were much better compared to basic ones. According to the recommendations, we managed to obtain the $y+$ parameter less than 50 in all of the computational grids used.

As a basic geometry for the research, we used a Pelton turbine, which operates with a head of $520 \mathrm{~m}$, a maximum flow rate of $1.5 \mathrm{~m}^{3} / \mathrm{s}$ and that has a nozzle diameter of $162 \mathrm{~mm}$. The shape of the water jet behind the basic deflector is presented in Fig. 2. The boundary between the two phases in a two-phase flow is well defined.

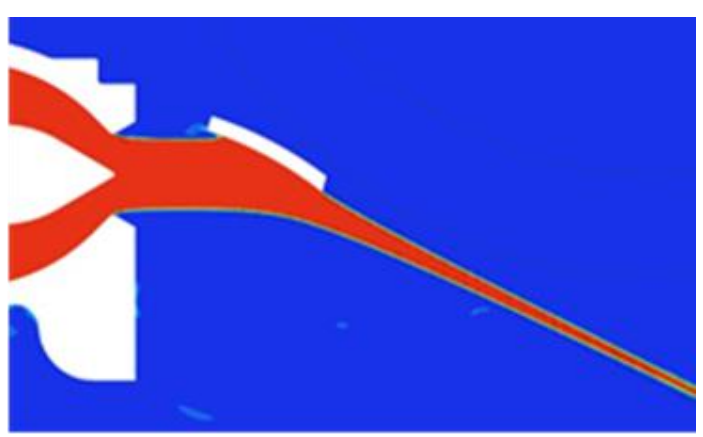

Fig. 2. Jet water volume fraction

For the position of the deflector at an angle of $18^{\circ}$ with respect to the open position, we have the entire reflection of the jet on the upper plate, without noticing the flow of water over the upper edge of the deflector. No back flow is observed anywhere. The distribution of pressure over the surface of the deflector is shown in Fig. 3.

The highest value of the pressure is observed at the highest point and it occupies a very small area. If we look to the left and right, we see a fairly even distribution of pressure, which is also expected with a quality jet. 


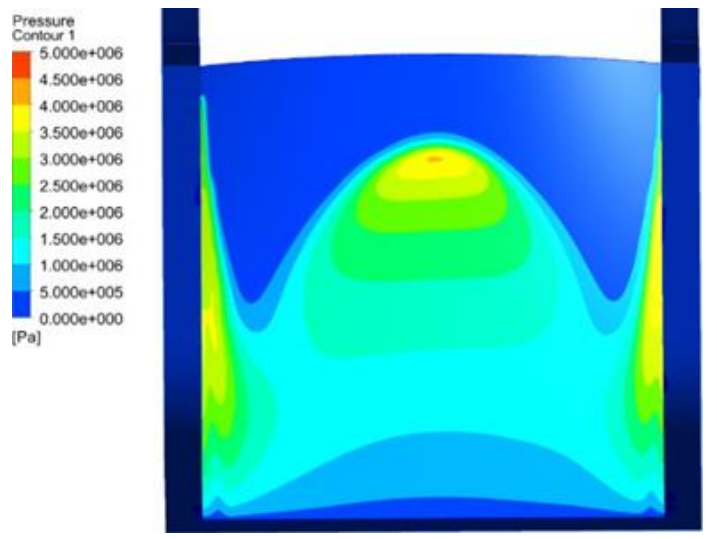

Fig. 3. Pressure distribution for final geometry

In the design process, the geometry of the deflector was without side walls, in order to shorten the computational time for huge number of calculations.

The numerical results obtained with the present study have not been verified experimentally, since similar calculations have already been performed to analyze the flow in the Pelton runner [13] and they possess a good match between the results. In the case of a numerical analysis of the flow in the jet deflector, we therefore assume that the accuracy of the calculation is at least on the same level, since in the preparation of all the parameters of the numerical analysis, we have considered the standards used in previous research [14]

\section{RESULT OF STRESS ANALYSIS}

For the preparation of the basic construction of the nozzle and jet-deflector with its components, the Inventor Autodesk software package is applied, while the stress analysis is performed using the Ansys Workbench package (Fig. 4) with imported geometry and previously calculated hydraulic loads (Fig. 5). For the purposes of the calculation, a numerical network was established i.e. the accounting domain divided into a number of elementary volume cells and appropriate surface zones. The total number of the elements that form the mesh is about 300.000 .

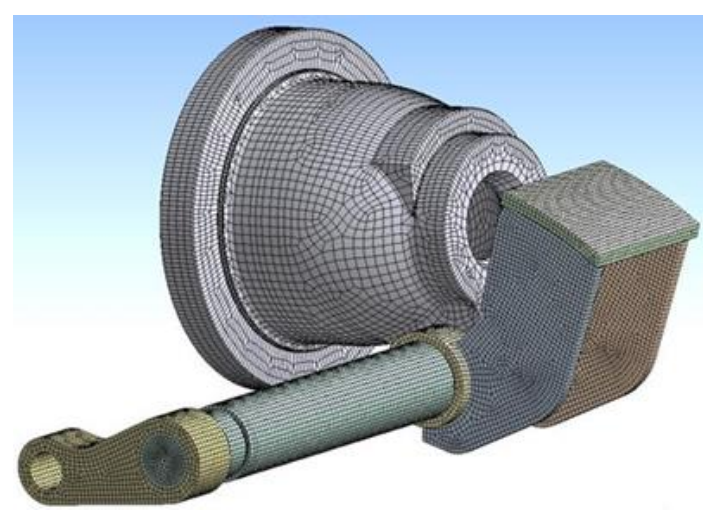

Fig. 4. Computational mesh
The basic construction of the nozzle was developed for the maximum pressure of $9 \mathrm{MPa}$. Fig. 5 shows the loads applied on the inner sides of the deflector plate including their values, two displacements at the end of the shaft, the cylindrical support and the fixed support on the nozzle.

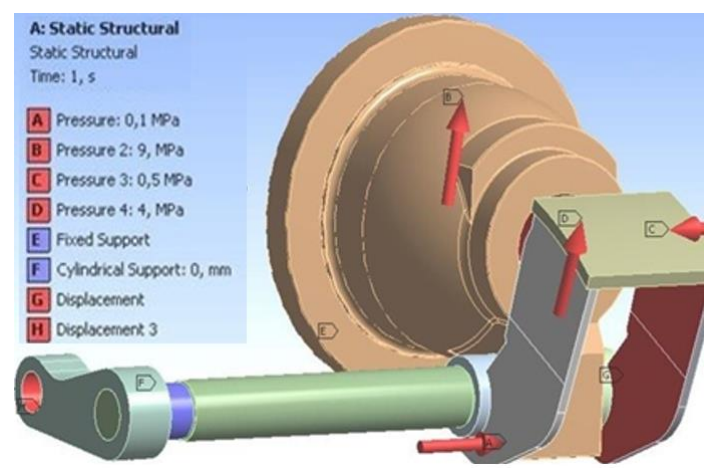

Fig. 5. Loads and contacts for stress analysis

In relation to this basic geometry, a strength analysis was conducted, and it is considered as starting construction with the following results for equivalent strain and total deformation; it is presented in Fig. 6.

Based on the images from the strength analysis, it may be concluded that equivalent strain of the construction is within the limit of $150 \mathrm{MPa}$ and the maximal total deformation of the deflector is $5.6 \mathrm{~mm}$.

In order to investigate the possibility of reducing the load (hydraulic forces and torque) and, following this, reducing strains, elastic extensions and total deformations of the jet-deflector, a variety of examinations were performed in relation to the shape of the deflector top plate, the angle of the placement and the closing angle of the deflector.

In the first step (Fig. 7), three additional geometries in the closed position of the deflector were analysed for angular placement of $-2^{\circ},+2^{\circ}$ and $+4^{\circ}$. The plate of the deflector is rotated around fixed axis-A.

Tab. 1. The values of hydraulic torque

\begin{tabular}{ccc}
\hline Position & Mh, kNm & Difference, \% \\
\hline$-2^{\circ}$ & 11,66 & $+3,2$ \\
\hline $0^{\circ}$ (basic) & 11,30 & 0 \\
\hline$+2^{\circ}$ & 10,67 & $-5,6$ \\
\hline$+4^{\circ}$ & 9,91 & $-12,3$ \\
\hline
\end{tabular}

Regarding the view of top plate's pressure distribution, all the positions are similar. The lowest value of the hydraulic torque of the top plate with angular position at $+4^{\circ}$ is $9.91 \mathrm{kNm}$; see Tab. 1 for the results. 
a)

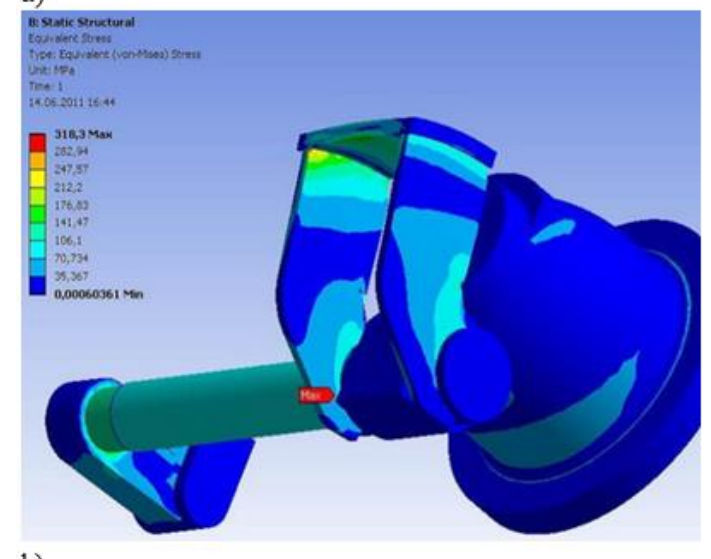

b)

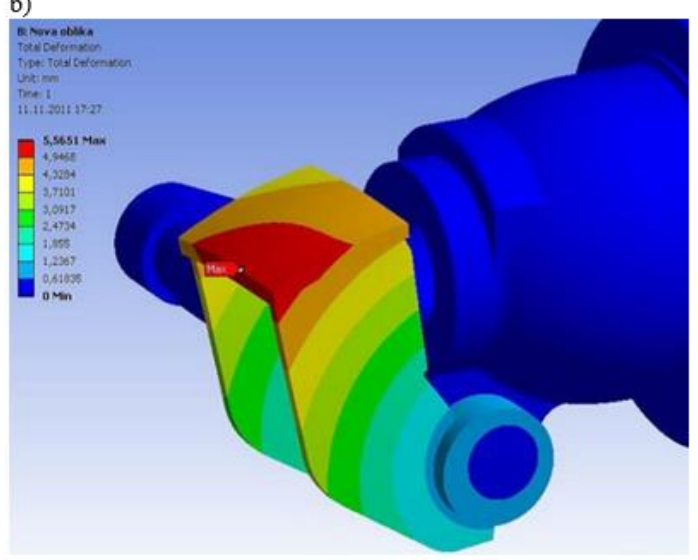

Fig. 6. a) equivalent strain (top) and b) total deformation (bottom)

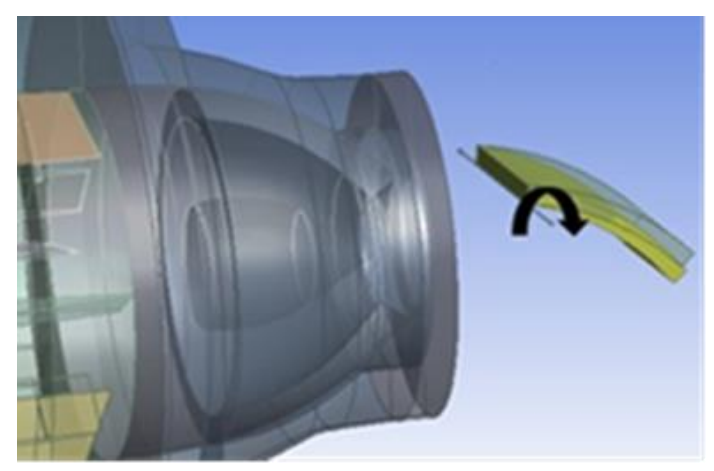

Fig. 7. Rotation around fixed axis

The higher angular position of the top plate, displaces the stagnation zone (high-pressure values area) to the inlet edge of the top plate. Proportionally to the higher angular position, the maximum pressure in is also found in the "stagnation zone".

The second step offers the possibility of reducing the torque and hydraulic forces, so the investigation leads to dissimilar shapes of the deflector top plate. With an alterable radius of the curve, three study cases were investigated. The basic (existing radius) $R=750$ $\mathrm{mm}$ increased the curve $R=400 \mathrm{~mm}$ and the flat plate (Fig. 8). a)

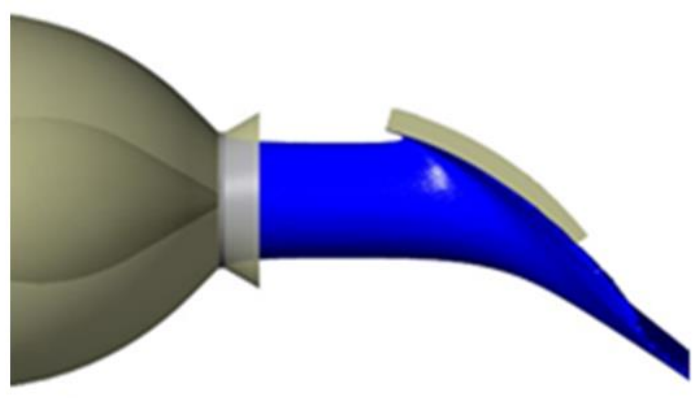

b)

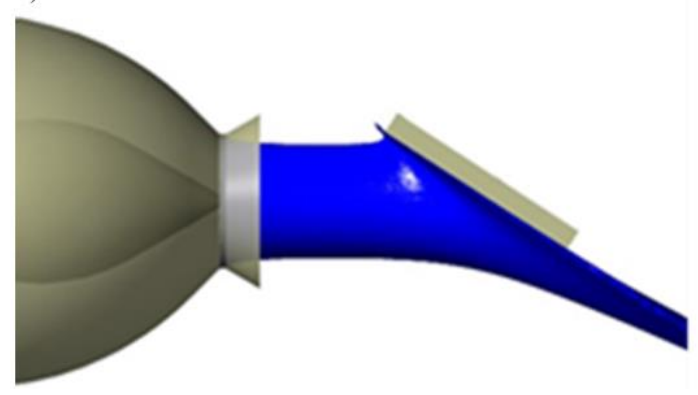

Fig. 8 . a) $R=400 \mathrm{~mm}$ (top) and b) flat plate (bottom)

As it can be observed based on the results of the analysis in the Tab. 2, higher maximal pressure values are the result of a higher radius expense. Also, as in the previous case with the top plate rotated, higher radius shifts the stagnation zone towards the leading edge of the plate, as we can see from the pressure distribution in Figure 3.

Tab. 2. Hydraulic forces and torque

\begin{tabular}{cccccc}
\hline Shape & $F_{x, \mathrm{~N}}$ & $F_{y}, \mathrm{~N}$ & $F_{z}, \mathrm{~N}$ & $F_{R}, \mathrm{~N}$ & $M_{h}, \mathrm{Nm}$ \\
\hline$R=400$ & 60,1 & 1,2 & 85,8 & 104,8 & 11,9 \\
\hline$R=750$ & 50,9 & 0,1 & 78,5 & 93,6 & 10,7 \\
\hline Flat & 43,4 & 0,8 & 66,1 & 79,0 & 6,9 \\
\hline
\end{tabular}

The basis for the modifications was an analysis of the pressure distribution along the longitudinal crosssection at the center of the jet deflector, as shown in Fig. 9. Based on this pressure distribution, forces and torques were calculated and the point of force action on the surface of the deflector was defined, which affects the final torque size.

The third step focuses on a modification of the input surface in order to reduce the high-pressure concentration on it. For this purpose, a totally distinct technical solution was chosen. It is rounded plate in a cross-section, with a part of a cylindrical surface with an appropriate radius. Three radii with convenient values (Fig. 10) were considered in the calculation. 


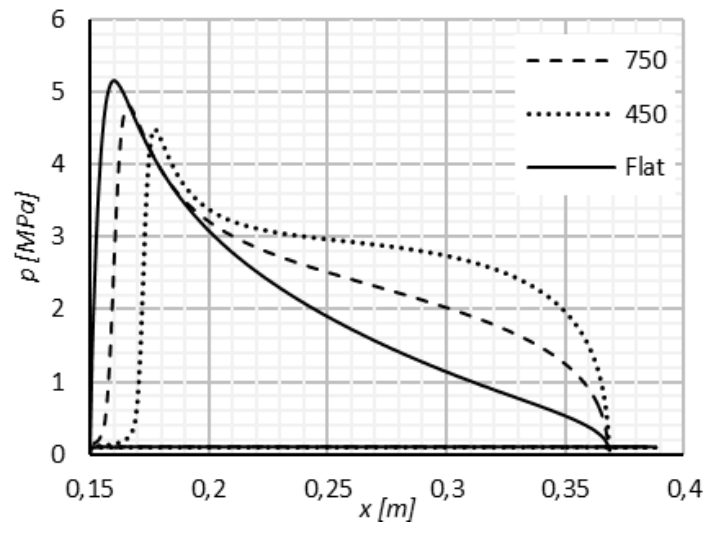

Fig. 9. Pressure distribution for different shapes

a)

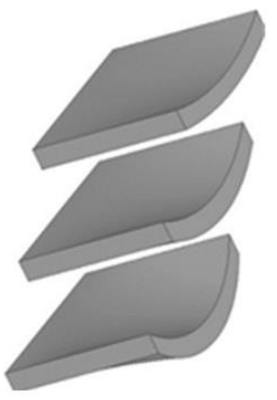

Fig. 10. Variable radius of the curve of the top plate: a) $R=350 \mathrm{~mm}, \mathrm{~b}) R=250 \mathrm{~mm}$ and c) $R=150 \mathrm{~mm}$

This "cylindrical surface" solution has some disadvantages. The exit of the water jet of the deflector has significant dissipation and, even more importantly, it requires an enormous distance between the runner and the nozzle due to the roundness of the top plate.

Taking all the previous results and conclusions into consideration, the final design solution fulfilled all the imperative criteria: the roundness of the plate on the entry surface with a $350 \mathrm{~mm}$ radius of the cylinder, which continuously modifies surface curvature into a linear shape toward the exit (Fig. 11).

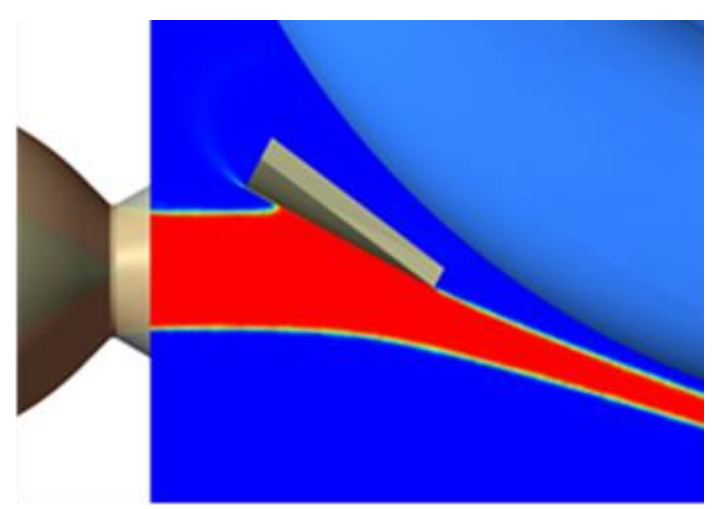

Fig. 11. Final design
Additional calculations of the hydraulic forces (Fig. 12) $F_{x}, F_{y}$, and $F_{z}$ and torque $M_{h}$ for three closing positions $12^{\circ}, 15^{\circ}$ and $18^{\circ}$ (basic solution) of the deflector were performed. The resultant force that affects the torque was obtained using equation $F^{\prime}=\left|F^{\prime}{ }_{x}-F^{\prime} y\right|$.

The plate that is attached to the deflector, on the same position of $18^{\circ}$ (the basic solution), ensures 20-25 $\mathrm{mm}$ of additional spare space between the blades of the runner and the trajectory of the exit water jet.

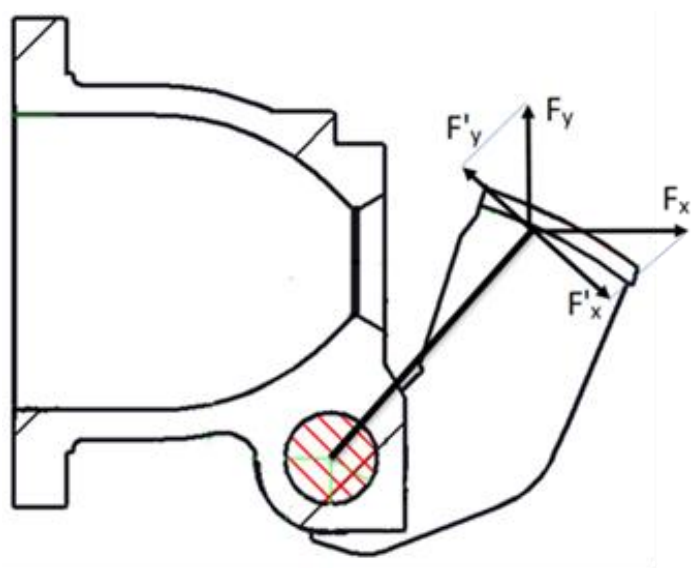

Fig. 12. Force components which influence on torque

In such newly occurring circumstances, the $15^{\circ}$ closing angle of the deflector matures in a technically optimal settlement and it allows an additional reduction of the duration of the hydrodynamic load.

The shape of the deflector plate is important not only to achieve optimal load forces and torques but also to position the deflector relative to the Pelton runner blades to avoid any unwanted contacts.

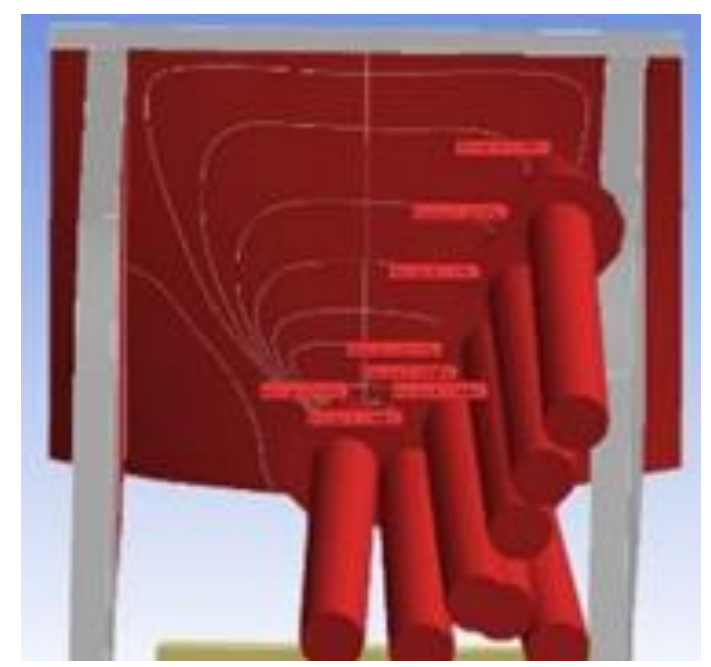

Fig. 13. Pressure distribution on deflector plate 
In the first instance, after optimizing and modelling the top plate of the deflector, a calculation of the results in the field of pressures on the plate was performed, obtained from the hydrodynamic calculation from Fig. 13.

The results obtained from the pressure diagram are applied along the surface of the top plate and the deflector side panels including all the other contacts of the jet-deflector and pressure on the nozzle.

Based on the final results, it may be concluded that, in general, the stresses of the elements of the construction are within the values of 75-98 MPa and the maximum peak value of $176 \mathrm{MPa}$. On the other hand, the basic construction has the maximum peak $318 \mathrm{MPa}$ and an average equivalent stress value ranges between 140-150 MPa.

Even if we do not consider the maximum peak values of these two constructions as reliable and precise enough for the final calculation, we can observe and conclude that there is the same decrease in an average stress level of the elements between the two constructions of ca. $40 \%$ (37-44) \%.

Stress reduction (SR) is calculated based on the results of the basic construction $\left(S_{b}\right)$ and the results of the optimized construction $\left(S_{o p t}\right)$ :

$$
\begin{gathered}
\mathrm{SR}=\frac{\mathrm{Sb}-\mathrm{Sopt}}{\mathrm{Sb}}= \\
(150-95) / 150=0,37=37 \% .
\end{gathered}
$$

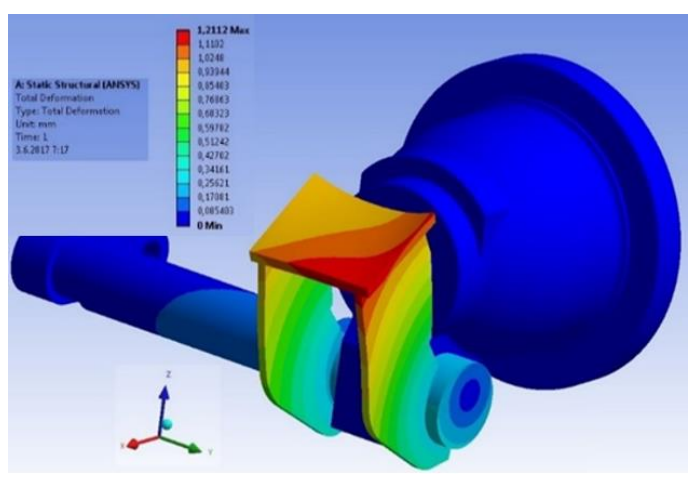

Fig. 14. Total deformation of the optimized deflector

It can be seen from the analysis of the results that there is a significant reduction in deformation (Fig. 14) in $\mathrm{X}$-axis $\mathrm{Y}$-axis and $\mathrm{Z}$-axis.

The reduction in total deformation (DR) is:

$$
\begin{gathered}
\mathrm{DR}=\frac{\mathrm{Db}-\mathrm{Dopt}}{\mathrm{Db}}= \\
(5,56-1,21) / 5,56=0,78=78 \% .
\end{gathered}
$$

Based on the results presented above, it can be seen that with an optimization of the geometry of the deflector, a significant reduction in the stresses and deformations was achieved, which is certainly the result of a significant reduction in the hydrodynamic load (hydrodynamic force and torque).

\section{CONCLUSIONS}

The jet deflector is not the most important part of the Pelton turbine, yet it has an important role in the case of a sudden stop of the turbine. The basic principles of the deflector's operation are quite simple; however, various complex problems can be encountered during the optimization process.

In such cases, the development of the deflector and its geometry contribute to a reduction of the loads and avoiding the oversized parts of the regulation system.

Starting from the force on the plate, through the torque that reached the hydraulic cylinder, the dimensions of the hydraulic cylinder, the power of the hydraulic aggregate and, finally, so do the size of the tank and the amount of oil that needed to allow a smooth operation of the whole system regulation. Apart from a reliable and faster operation of the deflector, these are just a part of the benefits of a stress analysis and reduced loads.

The paper presents the process of:

- a numerical analysis of the flow in Pelton turbine and an FEM analysis as the basis for the optimization process,

- the parameters which are used in the calculations were verified in the course of the previous research work,

- based on a detailed analysis of the pressure distribution and an analysis of the action of forces and torque, an optimized shape of the deflector can be obtained in a few steps, which cannot be actually optimal; however, the individual characteristics are considerably better compared to the initial geometry,

- all the components of the forces acting on the torque can be reduced, the point of adhesion of the force to the deflector can be moved and the load, deformation and torque on the axis of the deflector can significantly be reduced,

- the results obtained influence the operation of the servo motor that controls the deflector.

Based on the final results, a lot of useful information can be obtained for future research work. In future research, different types of optimization methods can be used, like multi-objective generic algorithms. Further research will also go in the direction of a self-closing deflector, which will need a servo motor only for the initial impulse of moving towards the jet.

\section{Nomenclature}

\section{Symbols}

F $\quad-$ force, N

$M$ - torque, $\mathrm{Nm}$

$p \quad-$ pressure, $\mathrm{Pa}$

$t \quad-$ time, s

$u \quad-$ velocity, $\mathrm{m} / \mathrm{s}$ 
$v_{t} \quad-$ eddy viscosity, $\mathrm{m}^{2} / \mathrm{s}$

$y^{+} \quad-$ non-dimensional distance

$\alpha, \beta \quad$ - fluid phase

$\kappa \quad-$ von Karman constant

$\rho \quad-$ density, $\mathrm{kg} / \mathrm{m}^{3}$

\section{Acronyms}

CFD - Computational Fluid Dynamics

CPU - Central Processing Unit

\section{References}

1. Li Ji-Qing, May Myat, Moe Saw, 2017, Fatigue Analysis of Simple and Advanced Hoop Pelton Turbine Buckets, American Scientific Research Journal for Engineering, Technology, and Sciences, Volume 29, No 1, pp 371-378.

2. Zhang Z., Muggli F., Parkinson E., Schärer C., 2000, Experimental investigation of a low head jet flow at a model nozzle of a Pelton turbine, International Seminar Wasserkraftanlagen, Wien.

3. Patel K., Patel B., Yadav M., and Foggia T., 2010, Development of Pelton turbine using numerical simulation, 25th IAHR Symposium on Hydraulic Machinery and Systems, 2010. doi:10.1088/17551315/12/1/012048

4. Zhang Z., Parkinson E., LDA application and the dualmeasurement-method in experimental investigations of the free surface jet at a model nozzle of a Pelton turbine, $20^{\text {th }}$ IAHR Symposium, Charlotte, North Carolina, USA

5. Mack R. and Moser W., 2002, Numerical Investigation of the Flow in a Pelton Turbine, Proc. of the XXI IAHR Symp. on Hydr. Machin. and Syst. (Lausanne, Switzerland).

6. Kvicinsky S., Kueny J.L., Avellan F., Parkinson E., 2002, Experimental and numerical analysis of free surface flows in a rotating bucket, Proceedings of the 21st IAHR Symposium, Lausanne, Switzerland.

7. Parkinson E., Neury C., Garcin H., Vullioud G. and Weiss T., 2005, Unsteady Analysis of a Pelton Runner with Flow and Mechanical Simulations, Hydro 2005, (Beljak, Austria).

8. Perrig A., Avellan F., Kueny J.L., Fahrat M., Parkinson E., 2006, Flow in a Pelton turbine bucket: numerical and experimental investigations, J. Fluids Eng.128. DOI: $10.1115 / 1.2170120$

9. Petley S. M, 2018, Numerical and Experimental Investigation of Flow in Horizontal Axis Pelton Turbines, Dissertation, Lancaster University, Engineering Department in collaboration with Gilbert Gilkes \& Gordon Ltd.

10. Bisen D., Shukla S., Sharma P.K., 2014, Optimization and Simulation of Hydro-Turbine Nozzle in Based on Ansys Analysis, International Journal of Advance Engineering and Research Development.

11. Barale D., Limbardi G., Arakerimath R. R., 2016, Modelling and Parametric Fluid Flow Analysis (CFD) and Effect on Convergent Nozzle Used in Pelton Turbine, Journal of Emerging Technologies and Innovative Research (JETIR).

12. Ansys CFX-Solver Theory Guide, 2015.

13. Jošt D., Meznar P. and Lipej A., 2010, Numerical prediction of Pelton turbine efficiency, 25th IAHR Symposium on Hydraulic Machinery and Systems. doi:10.1088/1755-1315/12/1/012080

14. Popovski B., 2017, Prediction of the geometry for jet deflector of Pelton turbine in respect of optimal design parameters, $\mathrm{PhD}$ Thesis (University Ss Cyril and Methodius, Faculty of Mechanical Engineering, Skopje, R. Macedonia).

\section{Biographical notes}

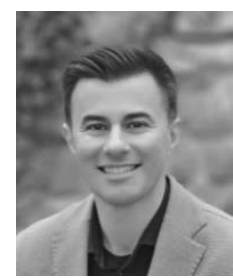

turbines.

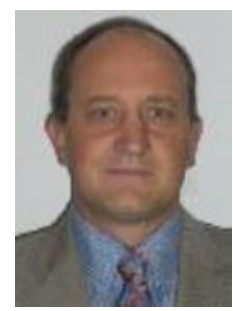

rotating machinery, optimization methods and design of different type of energetic machines.

Boro Popovski received his M.Sc. degree in Mechanical Engineering in 2011 and Ph.D in Mechanical Engineering in 2017 from University of Cyril and Methodius University in Skopje. His research work focuses on development of rotating machinery and design of different types of hydraulic

Andrej Lipej received his M.Sc. degree in Mechanics in 1990 and Ph.D in Mechanical Engineering in 1999 from University of Ljubljana. Currently he works as an associated professor at University of Novo mesto, Faculty of mechanical engineering. His scientific interests focus on problems of Computational Fluid Dynamics in 